Accepted for publication in The Astrophysical Journal

Preprint typeset using $\mathrm{LATE}_{\mathrm{E}} \mathrm{X}$ style emulateapj v. 12/16/11

\title{
DYNAMICAL MASSES FOR THE TRIPLE SYSTEM HD 28363 IN THE HYADES CLUSTER
}

\author{
Guillermo Torres, Robert P. Stefanik, and David W. Latham \\ Center for Astrophysics | Harvard \& Smithsonian, 60 Garden St., Cambridge, MA 02138, USA; gtorres@cfa.harvard.edu \\ Accepted for publication in The Astrophysical Journal
}

\begin{abstract}
The star HD 28363 in the Hyades cluster has been known for over a century as a visual binary with a period of $40 \mathrm{yr}$. The secondary is, in turn, a single-lined spectroscopic binary with a 21-day period. Here we report extensive spectroscopic monitoring of this hierarchical triple system that reveals the spectral lines of the third star for the first time. Combined with astrometric information, this makes it possible to determine the dynamical masses of all three stars. Only six other binaries in the Hyades have had their individual component masses determined dynamically. We infer the properties of the system by combining our radial velocity measurements with visual observations, lunar occultation measurements, and with proper motions from the Hipparcos and Gaia missions that provide a constraint on the astrometric acceleration. We derive a mass of $1.341_{-0.024}^{+0.026} M_{\odot}$ for the visual primary, and $1.210 \pm 0.021$ and $0.781 \pm 0.014 M_{\odot}$ for the other two stars. These measurements along with those for the other six systems establish an empirical mass-luminosity relation in the Hyades that is in broad agreement with current models of stellar evolution for the known age and chemical composition of the cluster.
\end{abstract}

\section{INTRODUCTION}

Relatively few binary or multiple systems in the nearby Hyades cluster have had the dynamical masses determined for their individual components. These are the essential ingredients for establishing the empirical massluminosity relation (MLR) in the cluster, which serves as a valuable test of current models of stellar evolution. Recent work by Torres (2019) has added one more system $(80 \mathrm{Tau})$ to the five classical binaries that have been used for this purpose in the Hyades. They are V818 Tau (McClure 1982; Schiller \& Milone 1987), $\theta^{2}$ Tau (Peterson et al. 1993; Tomkin et al. 1995), 51 Tau (Torres et al. 1997a), 70 Tau (Torres et al. 1997b), and $\theta^{1}$ Tau (Torres et al. 1997c).

The formal precision of these determinations is typically between $5 \%$ and $15 \%$, with the exception of the 5.6-day eclipsing binary V818 Tau, which has its masses measured to better than 1\%. Studies of the Hyades MLR in addition to those cited above include the papers by Lastennet et al. (1999) and by Lebreton et al. (2001), who also made a determination of the helium abundance of the cluster. More recent work on Hyades binaries has mostly dealt with improvements in the mass estimates of previously known systems.

In this paper, we present dynamical mass determinations for a new system, HD 28363 (HIP 20916 , WDS J04290+1610AB，HU 1080，ADS 3248 AB，vB 75; $V=5.56$ ), a visual binary with a 40 -yr period discovered in 1904 by Hussey (1905). The secondary is itself a spectroscopic binary with a period of about 21 days (Stefanik, \& Latham 1992; Mermilliod et al. 1994; Smekhov 1995), making the system a hierarchical triple. The 40-yr visual orbit with a semimajor axis slightly under 0!. 40 is reasonably well known (e.g., van den Bos 1956; Söderhielm 1999), and numerous radial-velocity measurements of the two visible stars have been collected mostly by Mermilliod et al. (2009) and Griffin (2012), which now cover both the inner and outer orbits well.
However, so far it has not been possible to determine the masses for all stars without making assumptions because the inner binary is only single-lined.

Here we report our own, extensive spectroscopic monitoring of the system for over three decades. We have detected the weak lines of the third star in most of our spectra and measured its velocities for the first time, enabling the masses to be determined for all three stars. This adds significantly to the small sample of Hyades binaries available to establish the empirical MLR in the cluster. Furthermore, we show that a global orbital solution combining our velocities and those of others with the visual observations and other astrometric constraints makes it possible to achieve much improved precision in the masses compared to most of the other systems.

We present our spectroscopic observations and the velocity measurements for the three stars in Section 2 The visual observations of the wide pair spanning more than a century are described in Section 3, together with other measurements and astrometric constraints from the Hipparcos and Gaia missions that measure the acceleration in the plane of the sky. Our orbital solution combining all measurements is reported in Section 4. The updated empirical MLR of the Hyades is discussed in Section 5 with a comparison to current models of stellar evolution. Our closing remarks are in Section 6.

\section{RADIAL VELOCITY MEASUREMENTS}

The history of the radial-velocity observations of HD 28363 has been documented by Griffin (2012), including the initial confusion as to which star of the visual pair was the 21-day spectroscopic binary. That issue was finally resolved by Smekhov (1995), who showed that it is the visual secondary, and published the first radial velocities for this star along with the elements of its spectroscopic orbit.

Griffin (2012) compiled a list of velocity measurements resulting from his own long-term monitoring of the system with several different instruments, as well as those 
from a similar program by Mermilliod et al. (2009). Together these 118 measurements span the interval from January of 1972 to January of 2010. An effort was made by Griffin to place them all on the same velocity system, so that effectively they may be considered as a single data set. The measurements are for the primary of the 40-yr visual pair ("star A") and the primary of the 21-day inner binary ("star Ba"). The secondary of the 21-day binary will be referred to in the following as "star $\mathrm{Bb}$ ". Where a brief designation is needed for the visual secondary, it will be called "star B". Our orbital analysis of Section 4 below will make use of the Griffin velocities as well as those of Smekhov (1995), which in addition to the 29 velocities for star Ba include 21 of star A. Several of the early measurements in the list by Griffin (2012) were considered by him to be unreliable, and we will reject them as well. His final data set has 111 velocities for star A and 116 for star Ba.

Our own spectroscopic monitoring of HD 28363 at the Center for Astrophysics (CfA), carried out as part of a large survey of the Hyades cluster, began in November of 1980 and continued until September of 2013. Observations were gathered with four different instruments. Spectra through November of 2007 were made with the CfA Digital Speedometers (DS; Latham 1992) on the $1.5 \mathrm{~m}$ Tillinghast reflector at the Fred L. Whipple Observatory (Mount Hopkins, AZ), the $1.5 \mathrm{~m}$ Wyeth reflector at the Oak Ridge Observatory (in the town of Harvard, MA), now closed, and the $4.5 \mathrm{~m}$-equivalent Multiple Mirror Telescope (also on Mount Hopkins) before its conversion to a monolithic $6.5 \mathrm{~m}$ mirror. These echelle instruments with a resolving power of $R \approx 35,000$ were equipped with intensified photon-counting Reticon detectors and recorded a single order $45 \AA$ wide centered at $5187 \AA$, featuring the $\mathrm{Mg}$ I b triplet. Signal-tonoise ratios for the 169 usable DS spectra range from about 20 to 83 per resolution element of $8.5 \mathrm{~km} \mathrm{~s}^{-1}$, although at the higher count levels flatfielding errors, rather than counts, are likely to dominate the uncertainty. An additional 28 spectra of higher quality were obtained using the Tillinghast Reflector Echelle Spectrograph (TRES; Szentgyorgvi \& Fürész 2007; Fürész 2008), a bench-mounted fiber-fed echelle instrument attached to the $1.5 \mathrm{~m}$ Tillinghast reflector, with a resolving power of $R \approx 44,000$. These spectra cover the wavelength range $3800-9100 \AA$ in 51 orders. For the order centered at about $5187 \AA$ the signal-to-noise ratios range between 81 and 284 per resolution element of $6.8 \mathrm{~km} \mathrm{~s}^{-1}$. Nightly sky exposures at dusk and dawn were used at each telescope to monitor the zero point of the DS instruments and place their velocities on a uniform system, which we refer to as the native CfA system. For TRES we relied on IAU standard stars to transfer the velocities to the same system as the DS.

We measured radial velocities using TRICOR, a threedimensional cross-correlation technique introduced by Zucker et al. (1995) that uses three templates, one for each component. We selected the templates from a large library of synthetic spectra based on model atmospheres by R. L. Kurucz, computed for the resolution of the DS and TRES instruments (see Nordström et al. 1994; Latham et al. 2002). These spectra span $300 \AA$ centered on the Mg I b triplet, which therefore includes the entire
$45 \AA$ order of the DS spectra. For the velocity determinations from TRES, we used only the $100 \AA$ order centered around $5187 \AA$, as experience shows that it contains most of the velocity information.

The two main template parameters affecting the velocities are the effective temperature $\left(T_{\text {eff }}\right)$ and rotational broadening ( $v \sin i$ when seen in projection). For the surface gravity parameter $(\log g)$ we adopted the value 4.5, which is appropriate for dwarf stars such as those in $\mathrm{HD} 28363$, and for the metallicity parameter $([\mathrm{Fe} / \mathrm{H}])$ we chose to use the solar value. This is within less than one step in our metallicity grid of the true composition of the Hyades $([\mathrm{Fe} / \mathrm{H}]=+0.18 \pm 0.03$; Dutra-Ferreira et al. 2016), which is sufficiently close for our purposes. After considerable experimentation, we found the best results were obtained with template temperatures of $6500 \mathrm{~K}$, $6000 \mathrm{~K}$, and $4500 \mathrm{~K}$ for stars $\mathrm{A}, \mathrm{Ba}$, and $\mathrm{Bb}$, respectively. The projected rotational velocity of star A has been reported by Griffin (2012) to be $v_{\mathrm{A}} \sin i=23.8 \pm 0.2 \mathrm{~km} \mathrm{~s}^{-1}$; we adopted $25 \mathrm{~km} \mathrm{~s}^{-1}$, which is the nearest value in our grid. Stars $\mathrm{Ba}$ and $\mathrm{Bb}$ were considered to be rotationally unbroadened, which in the case of $\mathrm{Ba}$ agrees with the assessment of Griffin (2012).

In a few of the weaker exposures from the DS, we were not able to measure the velocities of star $\mathrm{Bb}$ reliably, and only those for stars $\mathrm{A}$ and $\mathrm{Ba}$ are reported. The heliocentric radial velocities on the native CfA system for the three stars are listed in Table 1 along with their uncertainties. There are 169 for stars A and Ba and 158 for star $\mathrm{Bb}$. We also measured the flux ratios between pairs of stars using TRICOR, obtaining $\ell_{\mathrm{A}} / \ell_{\mathrm{Ba}}=2.079 \pm$ 0.095 and $\ell_{\mathrm{Bb}} / \ell_{\mathrm{Ba}}=0.049 \pm 0.007$ at a mean wavelength of $5187 \AA$.

Taken together, the CfA, Griffin (2012), and Smekhov (1995) data sets span a total of 36.5 years or about $90 \%$ of the visual binary period. The velocity measurements from all data sets are shown in Figure 1 and Figure 2 for the 40-yr outer orbit and 21-day inner orbit, respectively, along with our adopted model described later.

\section{ASTROMETRIC OBSERVATIONS}

\subsection{Visual measurements}

Since its discovery in 1904 the relative positions of HD 28363 (angular separations, $\rho$, and position angles, $\theta$ ) have been measured by visual observers about 130 times, not counting another dozen occasions in which the pair was not resolved. The most recent observation was obtained at the end of 2016. Measurements until about 1970 were made with filar micrometers, and most observations since were made using the speckle interferometry technique. A listing of all measurements was kindly provided to us by Brian Mason (U.S. Naval Observatory), extracted from the Washington Double Star Catalog (WDS), with the dates of observation having been uniformly converted from the traditional Besselian years to Julian years.

Most of these observations have no reported uncertainties. Their quality varies greatly and depends on many factors including the observing conditions, the telescope aperture used, and even the experience and disposition of the observer. Assigning realistic errors to any particular observation is non-trivial, and there is no unique way of doing this. Here we have chosen to divide the obser- 
Table 1

CfA Radial Velocities for HD 28363

\begin{tabular}{cccccrrr}
\hline \hline $\begin{array}{c}\text { HJD } \\
(2,400,000+)\end{array}$ & $\begin{array}{c}R V_{\mathrm{A}} \\
\left(\mathrm{km} \mathrm{s}^{-1}\right)\end{array}$ & $\begin{array}{c}\sigma_{\mathrm{A}} \\
\left(\mathrm{km} \mathrm{s}^{-1}\right)\end{array}$ & $\begin{array}{c}R V_{\mathrm{Ba}} \\
\left(\mathrm{km} \mathrm{s}^{-1}\right)\end{array}$ & $\begin{array}{c}\sigma_{\mathrm{Ba}} \\
\left(\mathrm{km} \mathrm{s}^{-1}\right)\end{array}$ & $\begin{array}{c}R V_{\mathrm{Bb}} \\
\left(\mathrm{km} \mathrm{s}^{-1}\right)\end{array}$ & $\begin{array}{c}\sigma_{\mathrm{Bb}} \\
\left(\mathrm{km} \mathrm{s}^{-1}\right)\end{array}$ & $\begin{array}{c}\text { Inner } \\
\text { Orbital Phase }\end{array}$ \\
\hline 44560.8550 & 44.85 & 0.60 & 66.37 & 0.44 & -13.56 & 5.52 & 0.49240 \\
Orbital Phase
\end{tabular}

Note. - (This table is available in its entirety in machine-readable form.)
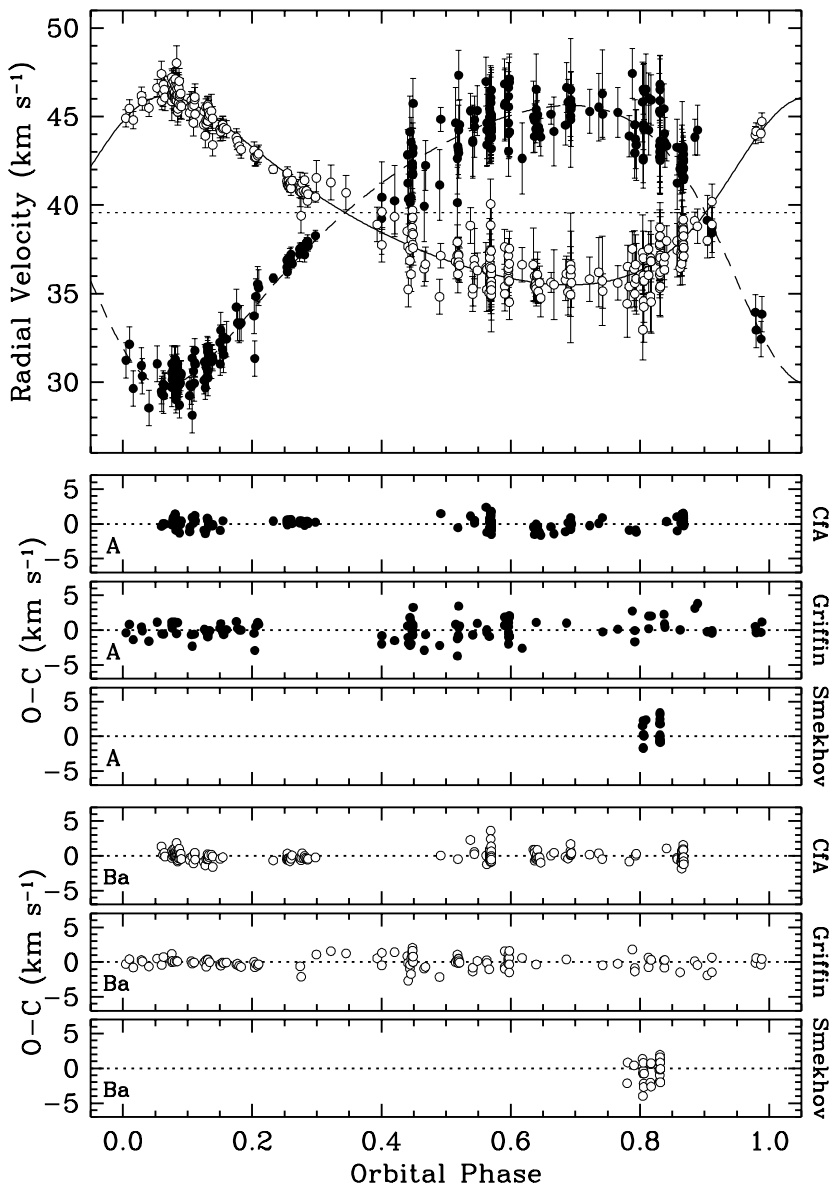

Figure 1. Top panel: Radial-velocity measurements of HD 28363 in the outer orbit, shown with our adopted model described in Section 4 The solid curve corresponds to the visual primary (star A), and the dashed one to the center of mass of the visual secondary (B). The points shown for the secondary are those of star Ba, corrected for motion in the inner orbit. We omit those of star $\mathrm{Bb}$ for clarity, as they show much larger scatter. The dotted line marks the center-of-mass velocity of the triple system. Measurements by Griffin (2012) and Smekhov (1995) have been adjusted for their respective zero-point offsets relative to CfA listed below in Table 5 Bottom panels: Residuals of the measurements for stars $\mathrm{A}$ and $\mathrm{Bb}$ from our adopted model, separately for the CfA, Griffin (2012), and Smekhov (1995) observations.

vations into groups by time period and by observational technique, and to assign uniform errors within each group iterating during the analysis described later to reach reduced $\chi^{2}$ values near unity within each group. For the position angles we specified the uncertainties in seconds of arc in the tangential direction, $\sigma_{\mathrm{t}}$, in order to explicitly take into account the dependence of the angular precision $\left(\sigma_{\theta}\right.$, expressed in degrees) on the angular separation:
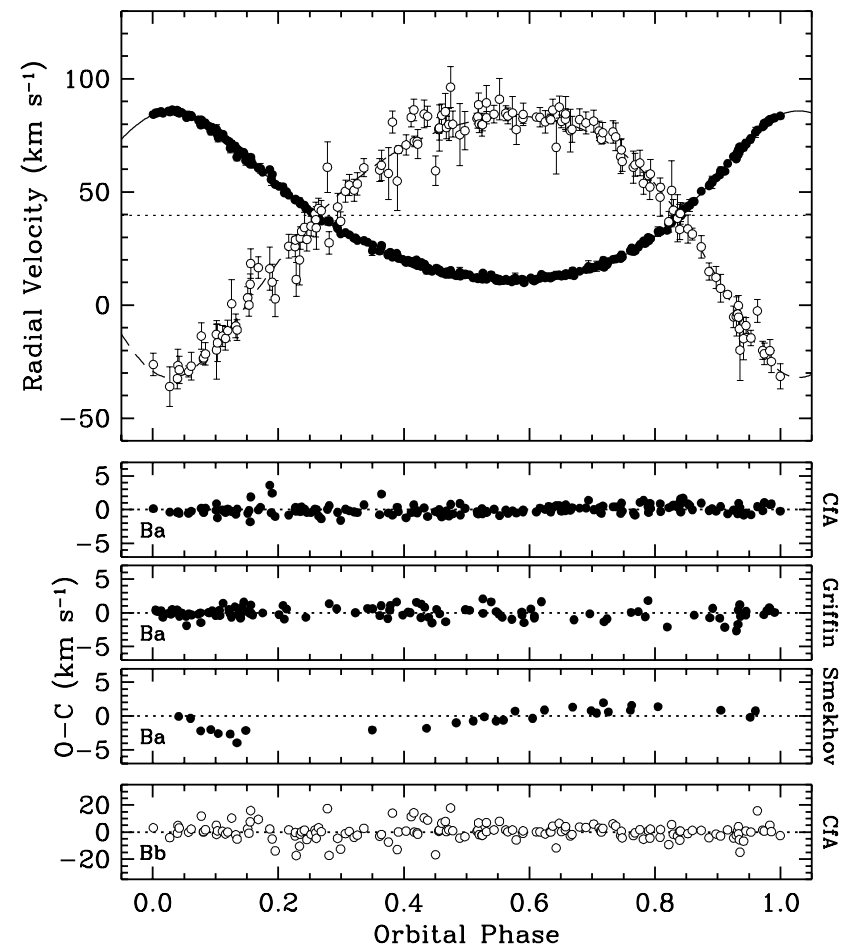

Figure 2. Top panel: Similar to Figure 1 for the inner orbit. The solid curve corresponds to star $\mathrm{Ba}$ and the dashed one to $\mathrm{Bb}$. The observations shown for stars $\mathrm{Ba}$ and $\mathrm{Bb}$ have had the motion in the outer orbit removed. The dotted line marks the center-ofmass velocity of the triple system. Bottom panels: Residuals of the measurements for the two stars, separately for the CfA, Griffin (2012), and Smekhov (1995) observations.

$\sigma_{\mathrm{t}}=\rho \sigma_{\theta}$. For observations prior to 1950 we adopted $\sigma_{\rho}=0$. .043 and $\sigma_{\mathrm{t}}=0$.'037; micrometer measurements between 1950 and 1970 were assigned $\sigma_{\rho}=0$.'035 and $\sigma_{\mathrm{t}}=0 . \prime 024$; more recent micrometer measurements had $\sigma_{\rho}=0.026$ and $\sigma_{\mathrm{t}}=0 . \prime 030$; and speckle observations received errors of $\sigma_{\rho}=0$.'004 and $\sigma_{\mathrm{t}}=0$ ". 0034 . In four cases (observations on 1923.93, 1925.10, 1927.06, and 1928.17 we found that the quadrants of the position angles needed to be reversed compared to the original records from the WDS. In a few other cases, the observations (mostly in angular separation) were rejected for being clearly different from others near in time.

In the end, we retained 126 measurements of the angular separation and 130 of the position angle of HD 28363 for the analysis below. These observations cover slightly more than two and a half cycles of the 40-yr orbit and are shown in Figure 3 together with our adopted model described later. A listing of the measurements is given in Table 2 . 
Table 2

Visual Observations of HD 28363

\begin{tabular}{ccccccccc}
\hline \hline Year & $\theta\left(^{\circ}\right)$ & $\sigma_{\mathrm{t}}\left({ }^{\prime \prime}\right)$ & $(O-C)_{\theta}\left({ }^{\circ}\right)$ & $(O-C)_{\theta} / \sigma_{\theta}$ & $\rho\left({ }^{\prime \prime}\right)$ & $\sigma_{\rho}\left({ }^{\prime \prime}\right)$ & $(O-C)_{\rho}\left(^{\prime \prime}\right)$ & $(O-C)_{\rho} / \sigma_{\rho}$ \\
\hline 1904.81 & 263.1 & 0.0238 & +4.26 & +1.41 & 0.44 & 0.0505 & -0.0118 & -0.23 \\
1906.96 & 256.6 & 0.0238 & -1.59 & -0.52 & 0.47 & 0.0505 & +0.0205 & +0.41 \\
1910.13 & 260.7 & 0.0238 & +3.59 & +1.04 & 0.40 & 0.0505 & +0.0065 & +0.13 \\
1910.132 & 260.7 & 0.0238 & +3.59 & +1.04 & 0.40 & 0.0505 & +0.0066 & +0.13 \\
1910.51 & 256.8 & 0.0238 & -0.15 & -0.04 & 0.42 & 0.0505 & +0.0379 & +0.75 \\
\hline
\end{tabular}

Note. - The position angles listed are the original ones; precession corrections were applied internally during the orbital analysis. The uncertainties listed in the table correspond to the $\sigma_{\rho}$ and $\sigma_{\mathrm{t}}$ values given in the text multiplied by the scaling factors $f_{\rho}$ and $f_{\theta}$ reported in Section 4 and represent the final errors used in the solution described there. Also listed are the residuals in angular separation and position angle normalized to their uncertainties $\sigma_{\rho}$ and $\sigma_{\theta}=\sigma_{\mathrm{t}} / \rho$. (This table is available in its entirety in machine-readable form.)
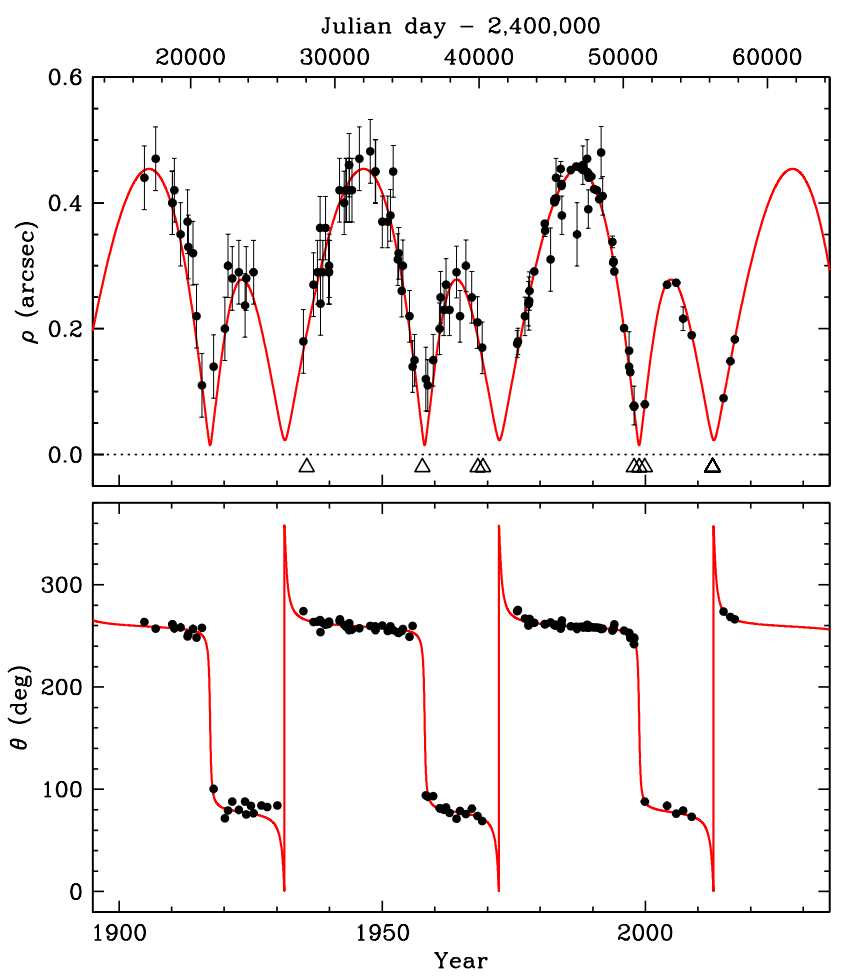

Figure 3. Angular separation and position angle measurements $(\rho, \theta)$ for HD 28363 as a function of time, with the adopted model described below in Section 4 Triangles at the bottom of the top panel indicate dates when the observers reported the binary was unresolved.

\subsection{Lunar occultations}

HD 28363 underwent a series of occultation events by the Moon between 1978 and 1980, many of which were recorded by observers, sometimes in several different filters. While these measurements are only onedimensional in nature and therefore do not provide as much information as the visual measurements, they are usually much more precise, they can have superior angular resolution, and typically also yield a good measurement of the magnitude difference between the components of a resolved binary. We, therefore, made use of these measurements in our orbital analysis of Section 4. Of the 11 recorded events included in the WDS listing we received, one by Peterson et al. (1981) was a neargrazing event and is best ignored, as described therein and as recommended also by (Evans 1984). Another by Richichi et al. (1999) that was not in the WDS listing did not resolve the pair; this may have been due to in-
Table 3

Lunar Occultation Observations of HD 28363

\begin{tabular}{cccccc}
\hline \hline Year & $\begin{array}{c}\psi \\
\left({ }^{\circ}\right)\end{array}$ & $\begin{array}{c}v \\
\left({ }^{\prime \prime}\right)\end{array}$ & $\begin{array}{c}\sigma_{v} \\
\left({ }^{\prime \prime}\right)\end{array}$ & $\begin{array}{c}(O-C)_{v} \\
\left({ }^{\prime \prime}\right)\end{array}$ & $(O-C)_{v} / \sigma_{v}$ \\
\hline 1978.7241 & 253.4 & 0.284 & 0.0054 & +0.0041 & +0.76 \\
1978.7241 & 254.5 & 0.258 & 0.0134 & -0.0227 & -1.69 \\
1978.7241 & 254.5 & 0.267 & 0.0134 & -0.0137 & -1.02 \\
1978.7242 & 241.2 & 0.2731 & 0.0089 & +0.0092 & +1.04 \\
1979.1728 & 279.4 & 0.2794 & 0.0121 & -0.0071 & -0.59 \\
1979.1728 & 286.3 & 0.2725 & 0.0030 & -0.0014 & -0.47 \\
1979.1728 & 287.1 & 0.281 & 0.0081 & +0.0088 & +1.10 \\
1980.0702 & 259.2 & 0.332 & 0.0188 & +0.0025 & +0.13 \\
1980.0704 & 262.5 & 0.3400 & 0.0107 & +0.0102 & +0.95 \\
1980.0705 & 258.9 & 0.3306 & 0.0016 & +0.0012 & +0.72 \\
\hline
\end{tabular}

Note. - The vector angles $\psi$ listed are the original ones; precession corrections were applied internally during the orbital analysis. The uncertainties in the vector separations $v$ are the original values multiplied by the scaling factor $f_{\text {occ }}$ reported in Section 4 and represent the final errors used in the solution described there. Residuals for the vector separations are listed in arc seconds and also normalized to their uncertainties.

strumental problems, but we note that it was taken precisely at periastron, when the predicted angular separation was only 15 milli-arc seconds. The remaining 10 measurements are of good quality and were retained for our analysis; they are listed in Table 3

\subsection{Hipparcos and Gaia}

HD 28363 has entries in both the Hipparcos Catalogue (ESA 1997) and in the second data release of the Gaia Catalogue (Gaia/DR2; Gaia Collaboration et al. 2018). The respective identifiers are HIP 20916 and Gaia/DR2 3312892757136810880 . The proper motions (p.m.) measured at each epoch (circa 1991.25 and 2015.5) differ significantly, which is a reflection of the acceleration in the plane of the sky due to the changing positions in the $40-\mathrm{yr}$ orbit. The p.m. difference therefore contains information on the orbit, and can be used to constrain it, as we do below. Brandt (2018) published a cross-calibrated catalog of Hipparcos and Gaia astrometry that facilitates this, and includes for each star a third p.m. measurement derived from the positional difference between the two original catalogs divided by the $\sim 24$ yr time baseline. As demonstrated by Brandt (2018) this last p.m. measure is practically independent of the other two and is particularly useful as it is considerably more precise.

We used all three p.m. determinations in this work to supplement the visual and lunar occultation observations and improve the orbital elements. At the time of the Hipparcos mission the angular separation between 
HD $28363 \mathrm{~A}$ and $\mathrm{B}$ was about $0^{\prime \prime} 4$, and the instrument was able to resolve the pair; the corresponding $\rho$ and $\theta$ measurements are included in the listing from the WDS. The published position and p.m. correspond to the primary, as indicated in the Catalogue. On the other hand, in 2015.5 the separation was only about 0 .' 12 , which is essentially at the resolution limit of Gaia (Gaia Collaboration et al. 2016). Values published in Gaia/DR2, therefore, correspond to the center of light rather than the primary. This important detail will be accounted for below.

Table 4 collects these measurements taken directly from the catalog of Brandt (2018), along with the uncertainties and correlation coefficients as listed there, and the average time of observation for each catalog in each coordinate. We include also the parallax measurements from the two missions, which we used as well to constrain our orbital fit as described below. The value for Hipparcos is the $60 / 40$ combination of the results from the re-reduction performed by van Leeuwen (2007) and from the original Hipparcos catalog (ESA 1997), following Brandt (2018), and includes an error inflation of 0.2 mas in quadrature as prescribed in his Eq.(18).

\section{ANALYSIS AND RESULTS}

We combined all radial-velocity and astrometric measurements into a global solution solving simultaneously for the elements of the inner and outer orbits, which were assumed to be non-interacting. The visual orbit (AB) has the following parameters: the period $P_{\mathrm{AB}}$, the angular semimajor axis $a_{\mathrm{AB}}^{\prime \prime}$, eccentricity parameters $\sqrt{e_{\mathrm{AB}}} \cos \omega_{\mathrm{B}}$ and $\sqrt{e_{\mathrm{AB}}} \sin \omega_{\mathrm{B}}$ (where $e_{\mathrm{AB}}$ is the eccentricity and $\omega_{\mathrm{B}}$ the argument of periastron for star $\mathrm{B}$, following the visual binary convention), the cosine of the inclination angle $\cos i_{\mathrm{AB}}$, the position angle of the ascending node $\Omega_{\mathrm{AB}}$ referred to the equinox J2000, and a reference time of periastron passage $T_{\mathrm{AB}}$. Precession corrections to J2000 were applied to all position angles from the visual and speckle measurements, as well as to the vector angles $\psi$ from the lunar occultations.

The spectroscopic elements of the outer orbit include the center-of-mass velocity $\gamma$ of the triple, and the velocity semiamplitudes of each visual component, $K_{\mathrm{A}}$ and $K_{\mathrm{B}}$. The inner spectroscopic orbit is described by its period $P_{\mathrm{B}}$, the corresponding eccentricity parameters $\sqrt{e_{\mathrm{B}}} \cos \omega_{\mathrm{Ba}}$ and $\sqrt{e_{\mathrm{B}}} \sin \omega_{\mathrm{Ba}}$ (with $\omega_{\mathrm{Ba}}$ being the argument of periastron for star $\mathrm{Ba}$, following the usual spectroscopic convention), the velocity semiamplitudes $K_{\mathrm{Ba}}$ and $K_{\mathrm{Bb}}$, and a reference time of periastron passage $T_{\mathrm{B}}$. Two extra parameters were included to account for possible zero-point offsets between the Griffin (2012) and Smekhov (1995) velocities and our own: $\Delta R V_{\mathrm{G}}$ and $\Delta R V_{\mathrm{S}}$. These shifts are to be added to those data sets in order to bring them onto the native CfA system. Though barely significant, light travel time corrections were computed at each step during the iterations and applied to the times of observation for the inner orbit. The maximum effect is about 0.047 days, corresponding to 0.0022 in phase.

The formalism for incorporating the Hipparcos, Gaia, and Hipparcos-Gaia proper motions $\left(\mu_{\alpha}^{*}\right.$ and $\left.\mu_{\delta}\right) \mathbb{1}$ was

${ }^{1}$ The $\mu_{\alpha}^{*}$ notation represents the p.m. in Right Ascension multiplied by $\cos \delta$. described in detail by Torres (2019) (see also Brandt 2018; Dupuy et al. 2019; Brandt et al. 2019), and we refer the reader to that work for details. Briefly, and using Hipparcos as an example, the p.m. in R.A. measured by that mission for the primary star $\left(\mu_{\alpha, \mathrm{H}, \mathrm{A}}^{*}\right)$ is simply that of the barycenter of the triple system $\left(\mu_{\alpha, 0}^{*}\right)$ plus a perturbation from the orbital motion of the primary around the barycenter: $\mu_{\alpha, \mathrm{H}, \mathrm{A}}^{*}=\mu_{\alpha, 0}^{*}+\Delta \mu_{\alpha, \mathrm{H}, \mathrm{A}}^{*}$. A similar expression can be written for the Declination component. The first term on the right is an adjustable parameter in our analysis (along with $\mu_{\delta, 0}$ ), and the second represents the change with time of the orbital position of the primary relative to the barycenter, at the average epoch $t_{\alpha, \mathrm{H}}$ of the Hipparcos measurement, as given in Table 4. Although both Hipparcos and Gaia observed HD 28363 over finite intervals of time of 2.5 and $1.7 \mathrm{yr}$, respectively, these are relatively short compared to the orbital period. We, therefore, assumed $\Delta \mu_{\alpha, \mathrm{H}, \mathrm{A}}^{*}$ (and an analogous perturbation term for Gaia) to be an instantaneous quantity and computed it as the time derivative of the orbital position.

Similarly, the Hipparcos-Gaia proper motion in R.A., $\mu_{\alpha, \mathrm{HG}}^{*}$, is that of the center of mass of the triple with an added term that incorporates the change in position between the two epochs:

$$
\mu_{\alpha, \mathrm{HG}}^{*}=\mu_{\alpha, 0}^{*}+\frac{\Delta \alpha_{\mathrm{G}}^{*}\left[t_{\alpha, \mathrm{G}}\right]-\Delta \alpha_{\mathrm{H}}^{*}\left[t_{\alpha, \mathrm{H}}\right]}{t_{\alpha, \mathrm{G}}-t_{\alpha, \mathrm{H}}} .
$$

Here the $\Delta \alpha^{*}$ quantities represent the position measured by the two missions relative to the barycenter at the mean epoch of each catalog and can be calculated from the orbital elements listed above. A similar equation holds for the Declination component $\mu_{\delta, \mathrm{HG}}$.

A complicating factor in the above is that Hipparcos measured the position and p.m. of the primary star, whereas Gaia measured the photocenter, as mentioned previously. The position and motion of star A can be computed easily enough from the orbital elements listed above, but doing the same for the center of light in the case of Gaia requires us to know the semimajor axis of the $\mathrm{AB}$ photocenter, $a_{\mathrm{ph}, \mathrm{AB}}^{\prime \prime}$. The relation between semimajor axis of the photocenter and that of the orbit of star $\mathrm{B}$ relative to $\mathrm{A}\left(a_{\mathrm{AB}}^{\prime \prime}\right)$ is $a_{\mathrm{ph}, \mathrm{AB}}^{\prime \prime}=a_{\mathrm{AB}}^{\prime \prime}\left(f_{\mathrm{AB}}-\beta_{\mathrm{G}}\right)$, in which $f_{\mathrm{AB}}$ is the fractional mass in the outer orbit and $\beta_{\mathrm{G}}$ is the fractional light in the Gaia bandpass. The mass fraction $f_{\mathrm{AB}} \equiv M_{\mathrm{B}} /\left(M_{\mathrm{A}}+M_{\mathrm{B}}\right)$ can be recast in terms of the orbital elements as $f_{\mathrm{AB}}=K_{\mathrm{A}} /\left(K_{\mathrm{A}}+K_{\mathrm{B}}\right)$. The fractional light $\beta_{\mathrm{G}}$ is unknown a priori but is constrained by the astrometry, so we included it as an additional adjustable parameter. This enables us to properly model the proper motions involving Gaia.

We carried out our analysis within a Markov Chain Monte Carlo (MCMC) framework using the emcee 2 code of Foreman-Mackey et al. (2013), which is a Python implementation of the affine-invariant MCMC ensemble sampler proposed by Goodman \& Weare (2010). We used 100 walkers with 10,000 links each, after discarding the burn-in. Priors for most variables were uniform, with those for $P_{\mathrm{AB}}$ and $a_{\mathrm{AB}}^{\prime \prime}$ being log-uniform. Convergence was checked by visual inspection of the chains, requiring

\footnotetext{
${ }^{2}$ https://emcee.readthedocs.io/en/v2.2.1/
} 
Table 4

Proper Motion and Parallax Information for HD 28363 from Gaia/DR2 and Hipparcos

\begin{tabular}{lccccc}
\hline \hline Source & $\mu_{\alpha}^{*}\left(\mathrm{mas} \mathrm{yr}^{-1}\right)$ & $\mu_{\delta}\left(\mathrm{mas} \mathrm{yr}^{-1}\right)$ & Corr & Average Epoch & $\pi(\mathrm{mas})$ \\
\hline HIP & $+89.35 \pm 1.53$ & $-24.08 \pm 0.95$ & -0.098 & $1991.26 / 1990.95$ & $20.62 \pm 1.27$ \\
Gaia-HIP & $+96.783 \pm 0.071$ & $-26.944 \pm 0.035$ & +0.325 & $\ldots$ & $\ldots$ \\
Gaia & $+117.11 \pm 0.62$ & $-22.74 \pm 0.39$ & -0.206 & $2015.58 / 2015.61$ & $20.74 \pm 0.18$ \\
\hline
\end{tabular}

Note. - Entries are taken from the acceleration catalog of Brandt (2018). $\mu_{\alpha}^{*}$ represents the p.m. in R.A. multiplied by the cosine of the Declination, and "Corr" is the correlation coefficient between the proper motions in R.A. and Dec. The "Average Epoch" is given separately for the p.m. measurements in R.A. and Dec. The Hipparcos parallax is a combination of results from the original mission and the re-reduction (ESA 1997; van Leeuwen 2007) (see text).

also a Gelman-Rubin statistic (Gelman \& Rubin 1992) less than 1.05 for all adjustable parameters.

The use of different kinds of observations requires careful relative weighting for a balanced solution. We handled this by incorporating multiplicative scaling factors for the uncertainties, which we adjusted simultaneously and self-consistently with the other orbital parameters (see Gregorv 2005). For the CfA velocities, the initial uncertainties for the three stars are those listed in Table 1. Our analysis used a different error inflation factor for each star, $f_{\mathrm{CfA} . \mathrm{A}}, f_{\mathrm{CfA} . \mathrm{Ba}}$, and $f_{\mathrm{CfA}, \mathrm{Bb}}$. The uncertainties for the Smekhov (1995) velocities were adopted as published, and the corresponding error inflation factors we introduced are $f_{\mathrm{S}, \mathrm{A}}$ and $f_{\mathrm{S}, \mathrm{Ba}}$. For the Griffin (2012) velocities we took into account the relative weighting of the observations recommended by the author for each star and each instrument and used the reported error of unit weight to calculate the formal uncertainties. The corresponding error scaling factors we then added are $f_{\mathrm{G}, \mathrm{A}}$ and $f_{\mathrm{G}, \mathrm{Ba}}$. We proceeded similarly with the angular separations and position angles from the visual measurements, and with the lunar occultations, adding three more error scaling factors $f_{\rho}, f_{\theta}$, and $f_{\text {occ }}$. Here $f_{\theta}$ represents the error inflation factor for the P.A. uncertainties in the tangential direction, $\sigma_{\mathrm{t}}$. The adopted priors for all of these factors were log-uniform. The uncertainties for the p.m. in Table 4 were taken at face value, and correlations between the R.A. and Dec. components were accounted for as described by Torres (2019) in computing the likelihood function.

We note that the combination of the visual and spectroscopic observations of HD 28363 yields the orbital parallax of the system. As Hipparcos and Gaia provided independent trigonometric parallaxes, we used those determinations as measurements with their corresponding uncertainties reported in Table 4 to further constrain the solution.

The results of the analysis are presented in Table 5 with an indication of the priors we used. The model has a total of 31 adjustable parameters. We report the mode of the corresponding posterior distributions for each parameter, along with the $68.3 \%$ confidence intervals. Table 6 lists several derived quantities computed directly from the chains of the corresponding adjustable parameters involved. From the fitted value of $\beta_{\mathrm{G}}$, we infer a magnitude difference between the two visual components in the Gaia bandpass of $\Delta G=0.739 \pm 0.070 \mathrm{mag}$.

The spectroscopic and visual observations together with the adopted model may be seen in Figures 1, 2, and 3 presented earlier. In Figure 2 we note that the residuals of the Smekhov (1995) velocities for star Ba
Table 5

Results of our MCMC Analysis for HD 28363

\begin{tabular}{|c|c|c|}
\hline Parameter & Value & Prior \\
\hline$P_{\mathrm{AB}}($ year $)$. & $40.752_{-0.034}^{+0.031}$ & {$[1,5]^{*}$} \\
\hline$a_{\mathrm{AB}}^{\prime \prime}(\operatorname{arcsec}) \ldots \ldots \ldots \ldots$ & $0.3749_{-0.0010}^{+0.0010}$ & {$[-3,2]^{*}$} \\
\hline$\sqrt{e_{\mathrm{AB}}} \cos \omega_{\mathrm{B}} \ldots \ldots \ldots \ldots$ & $+0.4154_{-0.0027}^{+0.0027}$ & {$[-1,1]$} \\
\hline$\sqrt{e_{\mathrm{AB}}} \sin \omega_{\mathrm{B}} \ldots \ldots \ldots \ldots$ & $-0.3811_{-0.0053}^{+0.0053}$ & {$[-1,1]$} \\
\hline $\cos i_{\mathrm{AB}} \ldots \ldots \ldots$ & $-0.0534_{-0.0014}^{+0.0012}$ & {$[-1,1]$} \\
\hline$\Omega_{\mathrm{AB}}($ degree $) \ldots$ & $78.395_{-0.060}^{+0.059}$ & {$[-180,+180]$} \\
\hline$T_{\mathrm{AB}}($ year $) \ldots$ & $1960.845_{-0.070}^{+0.070}$ & {$[1900,2000]$} \\
\hline$\gamma\left(\mathrm{km} \mathrm{s}^{-1}\right) \ldots$ & $+39.582_{-0.038}^{+0.038}$ & {$[+20,+60]$} \\
\hline$K_{\mathrm{A}}\left(\mathrm{km} \mathrm{s}^{-1}\right) \ldots \ldots \ldots \ldots$ & $7.927_{-0.063}^{+0.064}$ & {$[0,80]$} \\
\hline$K_{\mathrm{B}}\left(\mathrm{km} \mathrm{s}^{-1}\right) \ldots \ldots$ & $5.343_{-0.052}^{+0.057}$ & {$[0,80]$} \\
\hline$P_{\mathrm{B}}($ day $) \ldots \ldots$ & $21.254396_{-0.000025}^{+0.000024}$ & {$[20,23]$} \\
\hline$\sqrt{e_{\mathrm{B}}} \cos \omega_{\mathrm{Ba}}$ & $+0.4794_{-0.0015}^{+0.0015}$ & {$[-1,1]$} \\
\hline$\sqrt{e_{\mathrm{B}}} \sin \omega_{\mathrm{Ba}} \ldots$ & $-0.1530_{-0.0026}^{+0.0026}$ & {$[-1,1]$} \\
\hline$K_{\mathrm{Ba}}\left(\mathrm{km} \mathrm{s}^{-1}\right) \ldots$ & $37.245_{-0.055}^{+0.054}$ & {$[0,80]$} \\
\hline$K_{\mathrm{Bb}}\left(\mathrm{km} \mathrm{s}^{-1}\right) \ldots \ldots \ldots \ldots$ & $57.72_{-0.41}^{+0.44}$ & {$[0,80]$} \\
\hline$T_{\mathrm{B}}(\mathrm{HJD}-2,400,000) \ldots$ & $50828.045_{-0.016}^{+0.016}$ & {$[50800,50850]$} \\
\hline$\Delta R V_{\mathrm{G}}\left(\mathrm{km} \mathrm{s}^{-1}\right) \ldots$ & $-0.849_{-0.072}^{+0.072}$ & {$[-5,+5]$} \\
\hline$\Delta R V_{\mathrm{S}}\left(\mathrm{km} \mathrm{s}^{-1}\right) \ldots$ & $+0.52_{-0.24}^{+0.24}$ & {$[-5,+5]$} \\
\hline$\mu_{\alpha, 0}^{*}\left(\operatorname{mas~yr}^{-1}\right)$ & $+105.15_{-0.13}^{+0.13}$ & {$[+80,+140]$} \\
\hline$\mu_{\delta, 0}\left({\left.\operatorname{mas~} \mathrm{yr}^{-1}\right) \ldots}\right.$ & $-24.861_{-0.039}^{+0.040}$ & {$[-50,0]$} \\
\hline$\beta_{\mathrm{G}} \ldots \ldots \ldots \ldots$ & $0.336_{-0.014}^{+0.014}$ & {$[0.2,0.5]$} \\
\hline$f_{\mathrm{CfA}, \mathrm{A}} \cdots$ & $1.131_{-0.064}^{+0.075}$ & {$[-5,+3]^{*}$} \\
\hline$f_{\mathrm{CfA}, \mathrm{Ba}}$ & $1.581_{-0.095}^{+0.111}$ & {$[-5,+3]^{*}$} \\
\hline$f_{\mathrm{CfA}, \mathrm{Bb}}$ & $0.834_{-0.044}^{+0.052}$ & {$[-5,+3]^{*}$} \\
\hline$f_{\mathrm{G}, \mathrm{A}} \cdots$ & $1.073_{-0.066}^{+0.077}$ & {$[-5,+3]^{*}$} \\
\hline$f_{\mathrm{G}, \mathrm{Ba}} \cdots$ & $1.353_{-0.090}^{+0.105}$ & {$[-5,+3]^{*}$} \\
\hline$f_{\mathrm{S}, \mathrm{A}} \cdots$ & $2.84_{-0.40}^{+0.56}$ & {$[-5,+3]^{*}$} \\
\hline$f_{\mathrm{S}, \mathrm{Ba}}$ & $3.63_{-0.45}^{+0.62}$ & {$[-5,+3]^{*}$} \\
\hline$f_{\rho} \ldots$ & $1.175_{-0.071}^{+0.084}$ & {$[-5,+3]^{*}$} \\
\hline$f_{\theta} \ldots$ & $0.642_{-0.037}^{+0.043}$ & {$[-5,+3]^{*}$} \\
\hline$f_{\text {occ }} \ldots \ldots \ldots \ldots \ldots \ldots$ & $2.64_{-0.49}^{+0.85}$ & {$[-5,+3]^{*}$} \\
\hline
\end{tabular}

Note. - The values listed correspond to the mode of the posterior distributions, and the uncertainties represent the $68.3 \%$ credible intervals. Priors marked with an asterisk are log-uniform over the specified ranges; all others are uniform. 
Table 6

Derived Properties from our MCMC Analysis of HD 28363

\begin{tabular}{|c|c|}
\hline Quantity & Value \\
\hline$e_{\mathrm{AB}}$ & $0.3179_{-0.0024}^{+0.0024}$ \\
\hline$\omega_{\mathrm{B}}($ degree $) ..$ & $317.46_{-0.57}^{+0.57}$ \\
\hline$i_{\mathrm{AB}}($ degree $)$. & $93.060_{-0.071}^{+0.078}$ \\
\hline$a_{\mathrm{AB}}(\mathrm{au})$ & $17.689_{-0.094}^{+0.095}$ \\
\hline$a_{\mathrm{B}}^{\prime \prime}$ (mas). & $4.108_{-0.015}^{+0.015}$ \\
\hline$e_{\mathrm{B}} \ldots \ldots$ & $0.2532_{-0.0014}^{+0.0014}$ \\
\hline$\omega_{\mathrm{Ba}}$ (degree). & $342.30_{-0.31}^{+0.31}$ \\
\hline$i_{\mathrm{B}}^{\mathrm{a}}$ (degree). & $75.1_{-1.5}^{+1.6}$ \\
\hline$a_{\mathrm{B}}(\mathrm{au})$. & $0.1889_{-0.0011}^{+0.0011}$ \\
\hline$\pi_{\text {orb }}(\operatorname{mas}) .$. & $21.75_{-0.11}^{+0.11}$ \\
\hline Distance (pc). & $45.98_{-0.24}^{+0.23}$ \\
\hline$M_{\mathrm{A}}\left(M_{\odot}\right) \ldots \ldots$ & $1.341_{-0.024}^{+0.026}$ \\
\hline$M_{\mathrm{Ba}}\left(M_{\odot}\right) \ldots \ldots$ & $1.210_{-0.021}^{+0.021}$ \\
\hline$M_{\mathrm{Bb}}\left(M_{\odot}\right) \ldots \ldots$ & $0.781_{-0.014}^{+0.014}$ \\
\hline$M_{\mathrm{B}}\left(M_{\odot}\right) \ldots \ldots$ & $1.991_{-0.034}^{+0.034}$ \\
\hline$q_{\mathrm{AB}} \equiv M_{\mathrm{B}} / M_{\mathrm{A}}$ & $1.483_{-0.022}^{+0.022}$ \\
\hline$q_{\mathrm{B}} \equiv M_{\mathrm{Bb}} / M_{\mathrm{Ba}} \ldots \ldots$ & $0.6452_{-0.0048}^{+0.0048}$ \\
\hline$\Delta G(\mathrm{mag})$. & $0.739_{-0.070}^{+0.070}$ \\
\hline
\end{tabular}

Note. - The values listed correspond to the mode of the posterior distributions and the $68.3 \%$ credible intervals.

a Because the position angle of the ascending node is not known for the B orbit, the true inclination may also be on the opposite side of $90^{\circ}$, i.e., 104.9 .

show a clear sinusoidal pattern as a function of phase in the inner orbit, indicative of some systematic error not present in the other data sets. We examined this further by performing separate, single-lined orbital solutions for the inner orbit using the Ba velocities from CfA, Griffin (2012), and Smekhov (1995), all corrected in the same way for motion in the outer orbit using the parameters from the solution above. We found the three velocity semi-amplitudes $K_{\mathrm{Ba}}$ to be consistent with each other within uncertainties, so we chose to keep the Smekhov (1995) velocities for our global analysis. The residuals of the astrometric observations (visual and lunar occultation measurements) are given in Table 2 and Table 3 , in natural units and also normalized to the measurement uncertainties.

A representation of the outer orbit in polar coordinates is presented in Figure 4, showing it to be nearly edge-on $\left(i_{\mathrm{AB}} \approx 92^{\circ} .8\right)$. The inner orbit is also quite highly inclined. Table 6 lists it as $i_{\mathrm{B}} \approx 75^{\circ}$, but it could also be the symmetrical value relative to $90^{\circ}\left(105^{\circ}\right)$, given that we are missing information on $\Omega_{\mathrm{B}}$. Either way the two orbits cannot be exactly coplanar. The angular semimajor axis of the orbit of $\mathrm{B}$ is computed to be $a_{\mathrm{B}}^{\prime \prime} \approx 4.1$ mas. Careful re-examination of the epoch astrometry from the Hipparcos mission (transit observations) with the improved knowledge we now have of the orbit of B did not reveal the signature of that star, indicating those observations are not quite precise enough to detect the wobble

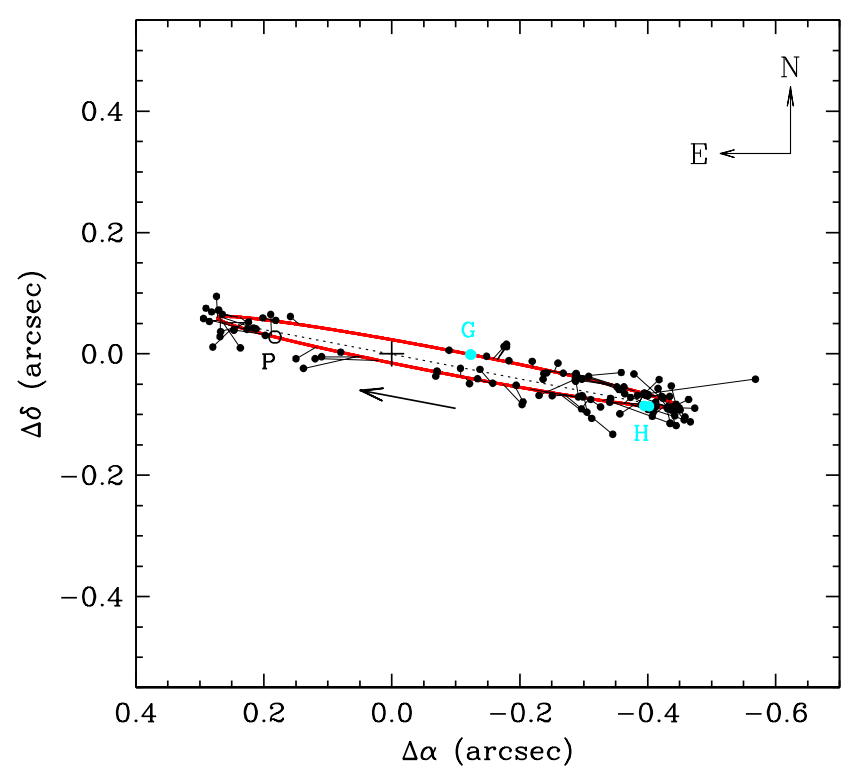

Figure 4. Visual observations and adopted model for the 40yr outer orbit in the plane of the sky. The plus sign marks the position of the primary. The direction of motion is clockwise, as indicated by the arrow. The dotted line is the line of nodes, and the open circle on the left labeled "P" indicates the location of periastron. Thin lines connect the observations with the predicted position along the orbit. For reference, we show also the position of the secondary at the time of the Hipparcos ("H") and Gaia ("G") measurements.

of stars $\mathrm{Ba}$ and $\mathrm{Bb}$. However, with a typical separation of the order of 4.1 mas spatial resolution of the B pair should be within reach of modern interferometers provided they have sufficient sensitivity. We estimate the apparent $K$-band magnitude of the B pair to be about 6.0 , and that of the individual components as $6.2(\mathrm{Ba})$ and $7.7(\mathrm{Bb})$. The angular separation of the $\mathrm{Ba}+\mathrm{Bb}$ pair can now be predicted accurately for any given time from the above elements; however, we cannot do the same for the position angle because the orientation of the line of nodes is unknown.

A comparison of our results with previously published orbital solutions for the visual pair and the inner binary by Smekhov (1995), Söderhjelm (1999), and Griffin (2012) is presented in Table 7.

\section{EMPIRICAL MASS-LUMINOSITY RELATION}

In order to make use of the mass determinations of HD 28363 from the previous section to place them on the empirical MLR of the Hyades, we require a measure of the apparent brightness of each star in the visual band. For this, we used the total $V$-band magnitude by Joner et al. (2006) $(V=6.565 \pm 0.004)$ and our spectroscopic light ratios $\ell_{\mathrm{A}} / \ell_{\mathrm{Ba}}$ and $\ell_{\mathrm{Bb}} / \ell_{\mathrm{Ba}}$ from Section 2, which correspond strictly to a mean wavelength of $5187 \AA$. To transform them to the $V$ band we appealed to synthetic spectra based on PHOENIX model atmospheres from the library of Husser et al. (2013) for the same temperatures, surface gravities, and metallicity adopted in our cross-correlation analysis. For each pair of stars, the predicted flux ratio as a function of wavelength was rescaled by adjusting the unknown ratio of the radii until we reproduced the light ratio at $5187 \AA$. We then integrated over the $V$ band to obtain $\left(\ell_{\mathrm{A}} / \ell_{\mathrm{Ba}}\right)_{V}=2.00 \pm 0.15$ and $\left(\ell_{\mathrm{Bb}} / \ell_{\mathrm{Ba}}\right)_{V}=0.072 \pm 0.015$. 
Table 7

Comparison of Orbital Solutions for HD 28363

\begin{tabular}{|c|c|c|c|c|}
\hline Parameter & Smekhov (1995) & Söderhielm (1999) & Griffin (2012) & This work \\
\hline \multicolumn{5}{|c|}{ Outer orbit } \\
\hline$P_{\mathrm{AB}}($ year $) \ldots \ldots \ldots \ldots$ & $40.38 \pm 0.01$ & 40.7 & $40.24 \pm 0.58$ & $40.752_{-0.034}^{+0.031}$ \\
\hline$a_{\mathrm{AB}}^{\prime \prime}(\operatorname{arcsec}) \ldots \ldots \ldots \ldots$ & $0.411 \pm 0.004$ & 0.39 & $\cdots$ & $0.3749_{-0.0010}^{+0.0010}$ \\
\hline$e_{\mathrm{AB}} \ldots \ldots \ldots \ldots \ldots \ldots$ & $0.39 \pm 0.01$ & 0.33 & $0.312 \pm 0.014$ & $0.3179_{-0.0024}^{+0.0024}$ \\
\hline$i_{\mathrm{AB}}($ degree $) \ldots \ldots \ldots \ldots$ & $92.5 \pm 0.4$ & 92 & $\cdots$ & $93.060_{-0.071}^{+0.078}$ \\
\hline$\omega_{\mathrm{B}}($ degree $) \ldots \ldots \ldots \ldots$ & $296.88 \pm 0.09$ & 311 & $315.0 \pm 3.0$ & $317.46_{-0.57}^{+0.57}$ \\
\hline$\Omega_{\mathrm{AB}}$ (degree). & $78.0 \pm 0.2$ & 78 & $\cdots$ & $78.395_{-0.060}^{+0.059}$ \\
\hline$T_{\mathrm{AB}}($ year $) \ldots \ldots \ldots \ldots$ & $1958.12 \pm 0.02^{*}$ & 1960 & $1961.15 \pm 0.25^{*}$ & $1960.845_{-0.070}^{+0.070}$ \\
\hline$\gamma\left(\mathrm{km} \mathrm{s}^{-1}\right) \ldots \ldots \ldots \ldots \ldots$ & $\cdots$ & $\cdots$ & $+40.50 \pm 0.06$ & $+39.582_{-0.038}^{+0.038}$ \\
\hline$K_{\mathrm{A}}\left(\mathrm{km} \mathrm{s}^{-1}\right) \ldots \ldots \ldots \ldots$ & $\cdots$ & $\cdots$ & $8.27 \pm 0.15$ & $7.927_{-0.063}^{+0.064}$ \\
\hline$K_{\mathrm{B}}\left(\mathrm{km} \mathrm{s}^{-1}\right) \ldots \ldots \ldots \ldots$ & $\cdots$ & $\cdots$ & $5.68 \pm 0.10$ & $5.343_{-0.052}^{+0.057}$ \\
\hline \multicolumn{5}{|c|}{ Inner orbit } \\
\hline$P_{\mathrm{B}}($ day $) \ldots \ldots \ldots$ & $21.253 \pm 0.001$ & $\cdots$ & $21.254259 \pm 0.000033$ & $21.254396_{-0.000025}^{+0.000024}$ \\
\hline$K_{\mathrm{Ba}}\left(\mathrm{km} \mathrm{s}^{-1}\right) \ldots \ldots \ldots \ldots$ & $37.04 \pm 0.13$ & $\cdots$ & $37.07 \pm 0.10$ & $37.245_{-0.055}^{+0.054}$ \\
\hline$e_{\mathrm{B}} \ldots \ldots \ldots \ldots \ldots \ldots$ & $0.268 \pm 0.005$ & $\ldots$ & $0.2553 \pm 0.0019$ & $0.2532_{-0.0014}^{+0.0014}$ \\
\hline$\omega_{\mathrm{Ba}}($ degree $) \ldots \ldots \ldots \ldots$ & $348.7 \pm 0.9$ & $\cdots$ & $341.5 \pm 0.6$ & $342.30_{-0.31}^{+0.31}$ \\
\hline$T_{\mathrm{B}}(\mathrm{HJD}-2,400,000) \ldots \ldots$ & $50828.16 \pm 0.09^{*}$ & $\cdots$ & $50828.001 \pm 0.030^{*}$ & $50828.045_{-0.016}^{+0.016}$ \\
\hline
\end{tabular}

Note. - No uncertainties were reported for the orbital elements of Söderhjelm (1999). Times of periastron passage marked with an asterisk are projected forward or backward by an integer number of cycles from the original times published to facilitate comparison with this work.

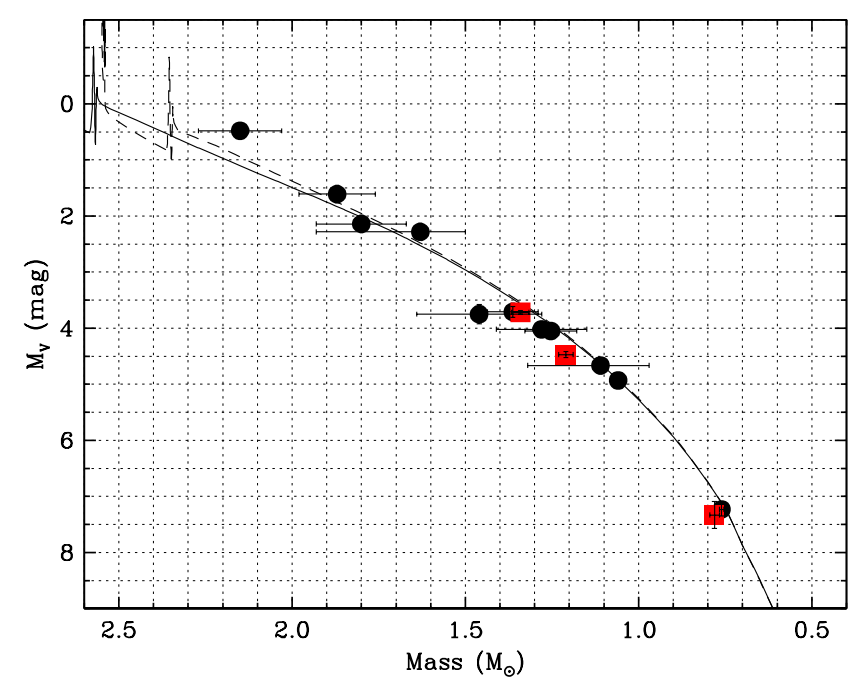

Figure 5. Empirical mass-luminosity relation in the Hyades. The stars in HD 28363 are shown with red squares. Measurements for the other six binary systems in the cluster with dynamical mass determinations (V818 Tau, $51 \mathrm{Tau}, 70 \mathrm{Tau}, \theta^{1} \mathrm{Tau}$, $\theta^{2} \mathrm{Tau}$, and $\left.80 \mathrm{Tau}\right)$ are taken from Table 3 of Torres (2019). The curves represent isochrones from the PARSEC series (Chen et al. 2014) for the known metallicity of the Hyades $([\mathrm{Fe} / \mathrm{H}]=+0.18$; Dutra-Ferreira et al. 2016) and ages of $625 \mathrm{Myr}$ (solid line) and 800 Myr (dashed).

With the orbital parallax from Table 6. the absolute visual magnitudes are then $M_{V}(\mathrm{~A})=3.717 \pm 0.032$, $M_{V}(\mathrm{Ba})=4.470 \pm 0.055$, and $M_{V}(\mathrm{Bb})=7.33 \pm 0.24$.

The mass and brightness of each HD 28363 component are shown in Figure 5 along with all other such measurements in the Hyades taken from Table 3 of Torres
(2019). The 14 individual mass estimates are broadly consistent with the stellar evolution models shown by the solid and dashed curves. These models correspond, respectively, to $625 \mathrm{Myr}$ and $800 \mathrm{Myr}$ isochrones from the PARSEC series by Chen et al. (2014), calculated for the metallicity of the Hyades. These models feature a modified temperature-opacity relation designed to provide a better fit to the mass-radius diagram of low-mass stars such as component Bb of HD 28363, and also yield improved fits to the lower main-sequence in the colormagnitude diagrams of clusters. We find that the new measurements for HD 28363 fall slightly below the models, though it is difficult to say whether this is because the masses are overestimated, the luminosities underestimated (perhaps from a bias in the orbital parallax in the upward direction), whether the models are too bright possibly due to missing opacities, or some combination thereof.

\section{CONCLUDING REMARKS}

The detection of the lines of star $\mathrm{Bb}$ in our spectra has enabled the determination of dynamical masses for all three members of the triple system HD 28363 for the first time. Without that information, it would only be possible to measure the mass of the primary star. The combination of our extensive spectroscopic monitoring and existing astrometric observations (visual and lunar occultation measurements, and p.m. and parallax measures from Hipparcos and Gaia) have enabled mass estimates with formal precisions better than $2 \%$ for the three stars. These are the most precise mass determinations for any Hyades binary with the exception of the eclipsing system V818 Tau. The K dwarf tertiary compo- 
nent of HD 28363 happens to be nearly identical in mass to the secondary of V818 Tau, and we find, reassuringly, that so is its brightness.

Dynamical mass determinations in the Hyades now populate the empirical MLR from about $0.76 M_{\odot}$ to $2.1 M_{\odot}$, though with a gap between $0.78 M_{\odot}($ HD 28363 $\mathrm{Bb})$ and $1.11 M_{\odot}(80 \mathrm{Tau} \mathrm{B})$, where the models show the largest change in slope (see Figure 5). Work is underway to identify additional cluster members amenable to mass determinations that may help fill in this gap.

Many of the spectroscopic observations of HD 28363 used here were obtained with the assistance of $\mathrm{M}$. Calkins, J. Caruso, P. Berlind, G. Esquerdo, E. Horine, R. Mathieu, J. Peters, and J. Zajac. We thank them all. We are also grateful to Brian Mason for providing a listing of the measurements of HD 28363 from the Washington Double Star Catalog. The referee is thanked as well for the prompt review of our manuscript and helpful comments. This research has made use of the SIMBAD and VizieR databases, operated at the CDS, Strasbourg, France, of NASA's Astrophysics Data System Abstract Service, and of the Washington Double Star Catalog maintained at the U.S. Naval Observatory. The work has also made use of data from the European Space Agency (ESA) mission Gaia (https://www.cosmos.esa.int/gaia), processed by the Gaia Data Processing and Analysis Consortium (DPAC, https://www.cosmos.esa.int/web/gaia/dpac/consortium Funding for the DPAC has been provided by national institutions, in particular the institutions participating in the Gaia Multilateral Agreement. The computational resources used for this research include the Smithsonian Institution's "Hydra" High Performance Cluster.

\section{REFERENCES}

Brandt, T. D. 2018, ApJS, 239, 31

Brandt, T. D., Dupuy, T. J., \& Bowler, B. P. 2019, arXiv e-prints, arXiv:1811.07285

Chen, Y., Girardi, L., Bressan, A., et al. 2014, MNRAS, 444, 2525

Dupuy, T. J., Brandt, T. D., Kratter, K. M., et al. 2019, ApJ, 871, L4

Dutra-Ferreira, L., Pasquini, L., Smiljanic, R., et al. 2016, A\&A, 585, A75

ESA, ed. 1997, ESA Special Publication, Vol. 1200, The Hipparcos and Tycho Catalogues

Evans, D. S. 1984, AJ, 89, 689

Foreman-Mackey, D., Hogg, D. W., Lang, D., \& Goodman, J. 2013, PASP, 125, 306

Foreman-Mackey, D. 2016, The Journal of Open Source Software, 24 , http://dx.doi.org/10.5281/zenodo.45906
Fürész, G. 2008, PhD thesis, Univ. Szeged, Hungary

Gaia Collaboration, Prusti, T., de Bruijne, J. H. J., et al. 2016, A\&A, 595, A1

Gaia Collaboration, Brown, A. G. A., Vallenari, A., et al. 2018, A\&A, 616, A1

Gelman, A., \& Rubin, D. B. 1992, Statistical Science, 7, 457

Goodman, J., \& Weare, J. 2010, Commun. Appl. Math. Comput. Sci., 5,65

Gregory, P. C. 2005, ApJ, 631, 1198

Griffin, R. F. 2012, Journal of Astrophysics and Astronomy, 33, 29

Høg, E., Fabricius, C., Makarov, V. V., et al. 2000, A\&A, 355, L27

Husser, T.-O., Wende-von Berg, S., Dreizler, S., et al. 2013, A\&A, $553, \mathrm{~A} 6$

Hussey, W. J. 1905, Lick Observatory Bulletin, 77, 116

Joner, M. D., Taylor, B. J., Laney, C. D., et al. 2006, AJ, 132, 111

Lastennet, E., Valls-Gabaud, D., Lejeune, T., et al. 1999, A\&A, 349,485

Latham, D. W. 1992, in IAU Coll. 135, Complementary

Approaches to Double and Multiple Star Research, ASP Conf. Ser. 32, eds. H. A. McAlister \& W. I. Hartkopf (San Francisco: ASP), 110

Latham, D. W., Stefanik, R. P., Torres, G., et al. 2002, AJ, 124, 1144

Lebreton, Y., Fernandes, J., \& Lejeune, T. 2001, A\&A, 374, 540

McClure, R. D. 1982, ApJ, 254, 606

Mermilliod, J.-C., Duquennoy, A., \& Mayor, M. 1994, A\&A, 283, 515

Mermilliod, J.-C., Mayor, M., \& Udry, S. 2009, A\&A, 498, 949

Nordström, B., Latham, D. W., Morse, J. A., et al. 1994, A\&A, 287, 33

Perryman, M. A. C., Brown, A. G. A., Lebreton, Y., et al. 1998, A\&A, 331, 81

Peterson, D. M., Baron, R. L., Dunham, E., et al. 1981, AJ, 86, 280

Peterson, D. M., Stefanik, R. P., \& Latham, D. W. 1993, AJ, 105, 2260

Richichi, A., Ragland, S., Calamai, G., et al. 1999, A\&A, 350, 491

Schiller, S. J., \& Milone, E. F. 1987, AJ, 93, 1471

Smekhov, M. G. 1995, Astronomy Letters, 21, 396

Söderhjelm, S. 1999, A\&A, 341, 121

Stefanik, R. P., \& Latham, D. W. 1992, IAU Colloq. 135: Complementary Approaches to Double and Multiple Star Research, 173

Stefanik, R. P., Latham, D. W., \& Torres, G. 1999, IAU Colloq. 170: Precise Stellar Radial Velocities, 354

Szentgyorgyi, A. H., \& Fürész, G. 2007, Precision Radial Velocities for the Kepler Era, in The 3rd Mexico-Korea Conference on Astrophysics: Telescopes of the Future and San Pedro Mártir, ed. S. Kurtz, RMxAC, 28, 129

Tomkin, J., Pan, X., \& McCarthy, J. K. 1995, AJ, 109, 780

Torres, G. 2019, ApJ, in press (arXiv:1908.03215)

Torres, G., Stefanik, R. P., \& Latham, D. W. 1997a, ApJ, 474, 256

Torres, G., Stefanik, R. P., \& Latham, D. W. 1997b, ApJ, 479, 268

Torres, G., Stefanik, R. P., \& Latham, D. W. 1997c, ApJ, 485, 167

van den Bos, W. H. 1956, Circular of the Union Observatory

Johannesburg 115, 281

van Leeuwen, F. 2007, Astrophysics Space Science Library, Vol.

350, Hipparcos, the New Reduction of the Raw Data (Berlin: Springer)

Zucker, S., Torres, G., \& Mazeh, T. 1995, ApJ, 452, 863 